\title{
Optimal universal and state-dependent quantum cloning
}

\author{
Dagmar Bruß, ${ }^{1,4}$ David P. DiVincenzo, ${ }^{2}$ Artur Ekert, ${ }^{1}$ Christopher A. Fuchs, ${ }^{3}$ Chiara Macchiavello,,${ }^{1,4}$ and John A. Smolin ${ }^{2}$ \\ ${ }^{1}$ Clarendon Laboratory, University of Oxford, Parks Road, Oxford OX1 3PU, United Kingdom \\ ${ }^{2}$ IBM Research Division, T.J. Watson Research Center, Yorktown Heights, New York 10598 \\ ${ }^{3}$ Bridge Laboratory 12-33, California Institute of Technology, Pasadena, California 91125 \\ ${ }^{4}$ ISI, Villa Gualino, Viale Settimio Severo 65, 10133 Torino, Italy \\ (Received 23 May 1997; revised manuscript received 11 November 1997)
}

\begin{abstract}
We establish the best possible approximation to a perfect quantum cloning machine that produces two clones out of a single input. We analyze both universal and state-dependent cloners. The maximal fidelity of cloning is shown to be $5 / 6$ for universal cloners. It can be achieved either by a special unitary evolution or by a teleportation scheme. We construct the optimal state-dependent cloners operating on any prescribed two nonorthogonal states and discuss their fidelities and the use of auxiliary physical resources in the process of cloning. The optimal universal cloners permit us to derive an upper bound on the quantum capacity of the depolarizing quantum channel. [S1050-2947(98)03303-4]
\end{abstract}

PACS number(s): 03.67.Hk, 03.65.-w, 89.70.+c

\section{INTRODUCTION}

A $1 \rightarrow 2$ quantum cloner is a quantum-mechanical machine that transforms a system described by some given pure state $|\psi\rangle$ together with some prescribed state into two systems, each with a state as "close" as possible to the given one. Specifically, a quantum cloner for quantum bits (qubits) is defined by an input qubit $|\psi\rangle$, a blank qubit $|0\rangle$, an ancillary system $A$ in a state $|X\rangle$ (if necessary), and a unitary transformation $U$ acting on all three of these, such that

$$
|\psi\rangle|0\rangle|X\rangle \rightarrow|\Psi\rangle=U|\psi\rangle|0\rangle|X\rangle
$$

and, after the interaction, the reduced density operators for the two qubits are identical, i.e., if $\rho_{1}=\operatorname{Tr}_{2, A}(|\Psi\rangle\langle\Psi|)$ and $\rho_{2}=\operatorname{Tr}_{1, A}(|\Psi\rangle\langle\Psi|)$, then $\rho_{1}=\rho_{2}$. In general, ideal quantum cloners (i.e., ones for which $\rho_{1}=\rho_{2}=|\psi\rangle\langle\psi|$ ) do not exist: Only if $|\psi\rangle$ is ensured to be drawn from a fixed orthogonal set can such a quantum cloner be constructed [1-4]. This situation, however, leads naturally to the question, "How close to ideal can a cloner be?" This can be explored both as a function of the sets from which the unknown state can be drawn and as a function of various notions of "closeness" to ideality. In this paper, we explore two such sets and optimality criteria.

We define a universal $1 \rightarrow 2$ quantum cloner as a quantum machine that takes as an input one qubit in a completely unknown quantum state $|\psi\rangle$ and generates at the output two qubits such that each of them is in a state described by the reduced density operator of the form $\rho=\eta|\psi\rangle\langle\psi|+(1$ $-\eta) \frac{1}{2} \mathbf{1}$. The parameter $\eta$ describes the shrinking of the original Bloch vector $\vec{s}$ corresponding to the density operator $|\psi\rangle\langle\psi|$, i.e., if $|\psi\rangle\langle\psi|=\frac{1}{2}(\mathbf{1}+\vec{s} \cdot \overrightarrow{\boldsymbol{\sigma}})$ then $\rho=\frac{1}{2}(\mathbf{1}+\eta \vec{s} \cdot \overrightarrow{\boldsymbol{\sigma}})$, where $\mathbf{1}$ is the $2 \times 2$ identity matrix and $\vec{\sigma}$ represents the set of Pauli matrices. In this case, we shall be interested in the best possible cloner with respect to the criterion of maximal $\eta$, that is, maximal "local" fidelity $F=\langle\psi|\rho| \psi\rangle=\frac{1}{2}(1+\eta)$ between input and output. This case is important because it reveals the overall effectiveness with which purely quantum information, embodied in a completely unknown quantum state, can be copied.

In some cases the original qubit may be prepared in a state that is selected from a known ensemble of states. In such cases we can design a state-dependent cloner that is optimal with respect to a given ensemble; here we will consider ensembles composed of only two nonorthogonal quantum states $|a\rangle$ and $|b\rangle$. Here the criterion of optimality is that of optimizing the "global fidelity" between input and output, i.e., to make the state $|\Psi\rangle$ given in Eq. (1) have the largest inner product possible with $|a\rangle|a\rangle$ or $|b\rangle|b\rangle$, depending upon the input state. This case is of some importance, for instance, because of the way it compares and contrasts with optimal eavesdropping schemes on two-state quantum cryptographic protocols [5].

The paper is organized as follows. In Sec. II A we discuss the performance of a universal quantum cloner, analyzing the role of the symmetry and isotropy conditions imposed on the system. The cloning transformation with the optimal local fidelity is derived by a constructive proof and is shown to coincide (modulo some phase factors) with the cloning machine proposed by Bužek and Hillery [6]. We then demonstrate in Sec. II B that universal quantum cloners can also be implemented via quantum-state teleportation [7]. This method results in the creation of two imperfect clones at two different locations by a combination of a shared threeparticle entanglement and public broadcasting. In Sec. III we relax the universality requirement and study state-dependent cloners. We derive the optimal cloning transformations with respect to two-state input ensembles. We also comment on the role of state-dependent cloners in quantum cryptography and show that the "local" and "global" fidelity criteria lead to distinct notions of cloning in the state-dependent case. Finally, in Sec. IV, as an application of these results, we relate the optimality of universal cloners to quantum channel capacity. All technical details of the optimality proofs are included in Appendix A (universal cloners) and Appendix B (state-dependent cloners). Appendix $\mathrm{C}$ details the calcula- 
tions required to compare the optimal state-dependent cloner to optimal eavesdropping in quantum cryptography $[8,9]$.

Let us point out again that in this paper we restrict our discussion only to $1 \rightarrow 2$ cloners. More general results will be presented elsewhere.

\section{UNIVERSAL QUANTUM CLONER}

In Sec. II A we derive the unitary transformation with optimum fidelity for a universal $1 \rightarrow 2$ quantum cloner. We then show in Sec. II B the possibility of establishing this cloning transformation via teleportation.

\section{A. Optimal universal quantum cloner}

In this subsection we find the optimum fidelity for a quantum cloner that is defined as a unitary transformation acting on two initial qubits (the one to be cloned in state $|\psi\rangle$ (or $\rho_{\psi}=|\psi\rangle\langle\psi|$ ) and the second one in a standard state $|0\rangle$ ) and an auxiliary system, also referred to as ancilla. We will impose the following conditions on a universal quantum cloner: (I). $\rho_{1}=\rho_{2}$ (symmetry), (II a) $\vec{s}_{1}=\eta_{\psi} \vec{s}_{\psi}$ (orientation invariance of the Bloch vector), and (II b) $F=\operatorname{Tr}\left(\rho_{\psi} \rho_{1}\right)=$ const. (isotropy). $\rho_{1}$ and $\rho_{2}$ represent the reduced density operators of the two output qubits

$$
\rho_{1}=\operatorname{Tr}_{2, A}[|\Psi\rangle\langle\Psi|]
$$

where $|\Psi\rangle$ is the global state at the output of the cloner and the partial trace is performed on the second copy and the ancilla's degrees of freedom, and analogously for $\rho_{2} . F$ is the fidelity of the cloner.

Let us comment on these three conditions. The first condition demands that the reduced density matrices of the two output states are the same. This is what we mean by symmetric cloning. The second condition requires that the Bloch vector of the original state $\psi$ does not change its direction but only its length: It shrinks by a factor $\eta_{\psi}$, indicating that the clones are not pure states, due to entanglement between themselves and the ancilla. The third condition requires that the cloner treats every state in the same way, i.e., the fidelity and thus the reduction factor $\eta$ does not depend on the input vector.

We will see in the following that conditions (II a) and (II b) are not independent: If conditions (I) and (II a) are satisfied (II b) holds automatically, i.e., symmetry plus orientation invariance implies isotropy. On the other hand, we notice that any transformation on a qubit (i.e., on a Bloch vector) can be decomposed into a transversal (rotation) and a longitudinal (rescaling) part. By demanding that the cloner treats all input states in the same way the Bloch vector of the original qubit can only be rescaled but not rotated, because a rotation has always two fixed points on the sphere ("hairy ball' theorem), so at least two states are transformed in a "special", way that contradicts the universality requirement.

Thus for a symmetric cloner the second and the third condition are equivalent. This is the reason that we called them (II a) and (II b).

We start from a general ansatz for the unitary transformation $U$ performed by the cloner and acting on the total Hilbert space $\mathcal{H}^{T}=\mathcal{H}^{2} \otimes H^{2} \otimes \mathcal{H}^{x}$, where $x$ is the dimension of the Hilbert space for the ancilla states:

$$
\begin{aligned}
U|0\rangle|0\rangle|X\rangle= & a|00\rangle|A\rangle+b_{1}|01\rangle\left|B_{1}\right\rangle \\
& +b_{2}|10\rangle\left|B_{2}\right\rangle+c|11\rangle|C\rangle, \\
U|1\rangle|0\rangle|X\rangle= & \widetilde{a}|11\rangle|\widetilde{A}\rangle+\widetilde{b}_{1}|10\rangle\left|\widetilde{B_{1}}\right\rangle \\
& +\widetilde{b}_{2}|01\rangle\left|\widetilde{B_{2}}\right\rangle+\widetilde{c}|00\rangle|\widetilde{C}\rangle .
\end{aligned}
$$

Here $|X\rangle$ denotes the initial state of the ancilla. Capital letters $A, B_{i}, C, \ldots$ refer to output ancilla states. We have not specified the dimension of the ancilla and we have not assumed any orthogonality relation to hold between $|A\rangle,\left|B_{i}\right\rangle, \ldots$. The only condition we are imposing on $|A\rangle,\left|B_{i}\right\rangle, \ldots$ is that they are normalized. In this way we do not restrict our argument leading to the optimum cloner to a certain dimension of the Hilbert space of the ancilla. From this general ansatz we can also draw conclusions about the existence of symmetric and isotropic quantum cloning without ancilla, which are discussed in Sec. III A.

Due to the unitarity of the cloning transformation, the coefficients $a, b_{i}, c, \ldots$, which are in general complex, must satisfy the normalization conditions

$$
\begin{aligned}
& |a|^{2}+\left|b_{1}\right|^{2}+\left|b_{2}\right|^{2}+|c|^{2}=1, \\
& |\widetilde{a}|^{2}+\left|\widetilde{b}_{1}\right|^{2}+\left|\widetilde{b}_{2}\right|^{2}+|\widetilde{c}|^{2}=1
\end{aligned}
$$

and the orthogonality condition

$a^{*} \widetilde{c}\langle A \mid \widetilde{C}\rangle+b_{2}^{*} \widetilde{b}_{1}\left\langle B_{2} \mid \widetilde{B_{1}}\right\rangle+b_{1}^{*} \widetilde{b}_{2}\left\langle B_{1} \mid \widetilde{B_{2}}\right\rangle+c^{*} \widetilde{a}\langle C \mid \widetilde{A}\rangle=0$.

We now impose the constraints (I) and (II) to satisfy the symmetry and the isotropy properties. We define the free phases for the coefficients as $a=|a| e^{i \delta_{a}}, \tilde{a}=|\tilde{a}| e^{i \delta_{\tilde{a}}}$ and analogously for the other coefficients. From imposing the symmetry condition we find that our ansatz (3) and (4) has to fulfill the relations

$$
\begin{gathered}
\left|b_{1}\right|=\left|b_{2}\right|, \quad\left|\widetilde{b}_{1}\right|=\left|\widetilde{b}_{2}\right|, \\
\left|\left\langle B_{1} \mid \widetilde{B_{2}}\right\rangle\right|=\left|\left\langle B_{2} \mid \widetilde{B_{1}}\right\rangle\right|, \quad\left|\left\langle B_{1} \mid \widetilde{B_{1}}\right\rangle\right|=\left|\left\langle B_{2} \mid \widetilde{B_{2}}\right\rangle\right|
\end{gathered}
$$

and

$$
a b_{1}^{*}\left\langle B_{1} \mid A\right\rangle+c^{*} b_{2}\left\langle C \mid B_{2}\right\rangle=a b_{2}^{*}\left\langle B_{2} \mid A\right\rangle+c^{*} b_{1}\left\langle C \mid B_{1}\right\rangle,
$$

and the same as Eq. (8) for the tilded coefficients and ancilla states. Moreover,

$$
\begin{aligned}
& \widetilde{b}_{1}^{*} a\left\langle\widetilde{B}_{1} \mid A\right\rangle+\widetilde{a}^{*} b_{1}\left\langle\widetilde{A} \mid B_{1}\right\rangle=\widetilde{b}_{2}^{*} a\left\langle\widetilde{B}_{2} \mid A\right\rangle+\widetilde{a}^{*} b_{2}\left\langle\widetilde{A} \mid B_{2}\right\rangle, \\
& b_{1}^{*} \widetilde{c}\left\langle B_{1} \mid \widetilde{C}\right\rangle+c^{*} \widetilde{b}_{1}\left\langle C \mid \widetilde{B_{1}}\right\rangle=b_{2}^{*} \widetilde{c}\left\langle B_{2} \mid \widetilde{C}\right\rangle+c^{*} \widetilde{b}_{2}\left\langle C \mid \widetilde{B_{2}}\right\rangle .
\end{aligned}
$$

We will call $\left|b_{1}\right|=\left|b_{2}\right|=|b|$ and $\left|\widetilde{b}_{1}\right|=\left|\widetilde{b}_{2}\right|=|\widetilde{b}|$ from now on.

Let us now look into the constraints following from imposing condition (II a). Orientation invariance of the Bloch vector $\vec{s}$ means that all its components shrink by the same ratio $\eta_{\psi}$ : 


$$
\frac{s_{1_{x}}}{s_{\psi_{x}}}=\frac{s_{1_{y}}}{s_{\psi_{y}}}=\frac{s_{1_{z}}}{s_{\psi_{z}}}=\eta_{\psi}
$$

Using the unitary transformation (3) and (4) and imposing condition (10) we find the constraints (i) $|a|^{2}-|c|^{2}=|\widetilde{a}|^{2}$ $-|\widetilde{c}|^{2}, \quad$ (ii) $\quad|a|^{2}-|c|^{2}=\operatorname{Re}\left[\widetilde{b}_{1}^{*} a\left\langle\widetilde{B}_{1} \mid A\right\rangle+\widetilde{a}^{*} b_{1}\left\langle\widetilde{A} \mid B_{1}\right\rangle\right]$, (iii) $\operatorname{Im}\left[\widetilde{b}_{1}^{*} a\left\langle\widetilde{B}_{1} \mid A\right\rangle+\widetilde{a}^{*} b_{1}\left\langle\widetilde{A} \mid B_{1}\right\rangle\right]=0$, (iv) $b_{1}^{*} \widetilde{c}\left\langle B_{1} \mid \widetilde{C}\right\rangle$ $+c^{*} \widetilde{b}_{1}\left\langle C \mid \widetilde{B_{1}}\right\rangle=0, \quad(\mathrm{v}) \quad b_{2}^{*} a\left\langle B_{2} \mid A\right\rangle+c^{*} b_{1}\left\langle C \mid B_{1}\right\rangle=0$, (vi) $\quad \widetilde{b}_{2}^{*} \widetilde{a}\left\langle\widetilde{B_{2}} \mid \widetilde{A}\right\rangle+\widetilde{c}^{*} \widetilde{b_{1}}\left\langle\widetilde{C} \mid \widetilde{B_{1}}\right\rangle=0, \quad$ (vii) $\quad \widetilde{c}^{*} a\langle\widetilde{C} \mid A\rangle$ $-\widetilde{a}^{*} c\langle\widetilde{A} \mid C\rangle=0$, and $(1 \leftrightarrow 2)$.

Here the notation $1 \leftrightarrow 2$ indicates that as a result of the symmetry condition the same set of constraints has to hold for exchange of the indices 1 and 2 .

Inserting constraints (i) and (vii) into the explicit form for the ratio $\eta_{\psi}$, we find easily that $\eta_{\psi}$ is a constant, i.e., independent of the input state. Thus, as mentioned before, we find that conditions (II a) and (II b) are not independent: after imposing condition (I), condition (II b) is automatically satisfied when (II a) holds. Therefore, any symmetric cloner that does not rotate the initial state is isotropic.

The explicit form of the reduction factor $\eta$ is

$$
\eta=|a|^{2}-|c|^{2}
$$

which we want to maximize. The fidelity

$$
F=\operatorname{Tr}\left(\rho_{1}|\psi\rangle\langle\psi|\right)=\frac{1}{2}\left(1+\vec{s}_{1} \cdot \vec{s}_{\psi}\right),
$$

which for the symmetric isotropic cloner is related to the reduction factor as

$$
F=\frac{1}{2}(1+\eta)
$$

is maximized as well.

The maximization of the fidelity is carried out using the Lagrange multiplier method, which takes into account the constraints imposed on the cloning transformation due to the unitarity, symmetry, and isotropy conditions. Here we have also required the unitary transformation to be symmetric under exchange $|0\rangle \leftrightarrow|1\rangle$ which leads to $|a|=|\widetilde{a}|,|b|$ $=|\tilde{b}|$, and $\quad|c|=|\widetilde{c}|$. The explicit optimization procedure is reported in Appendix A.

The idea is to use the Lagrange multiplier technique and some knowledge about the coefficients $a$ and $b_{i}$ to find the best value for $|c|$. Then we use constraint (ii) to find the optimum value of $|a|$ that gives us $|b|$ via the normalization condition.

The results are

$$
|c|=0, \quad|a|=\sqrt{\frac{2}{3}}, \quad|b|=\sqrt{\frac{1}{6}} .
$$

Here $|c|=0$ can be understood intuitively because $c$ is the coefficient for the state $|11\rangle$ that is maximally remote from the ideal output state $|00\rangle$ in Eq. (3).

Thus we find that the shrinking factor $\eta$ of the optimum symmetric isotropic cloner is

$$
\eta=\frac{2}{3}
$$

corresponding to the optimum cloning fidelity

$$
F=\frac{5}{6}
$$

As shown in Appendix A, the class of unitary transformations for the optimal symmetric and isotropic cloner is given by

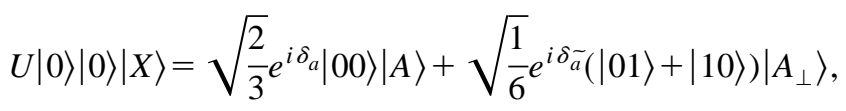

$U|1\rangle|0\rangle|X\rangle=\sqrt{\frac{2}{3}} e^{i \delta \tilde{a}}|11\rangle\left|A_{\perp}\right\rangle+\sqrt{\frac{1}{6}} e^{i \delta_{a}(|01\rangle+|10\rangle)|A\rangle,}$

where $\left\langle A \mid A_{\perp}\right\rangle=0$.

We can realize this transformation with two-dimensional ancilla states, e.g., $|A\rangle=|0\rangle,\left|A_{\perp}\right\rangle=|1\rangle$, or any other orientation of $|A\rangle$. These possibilities are different from each other with respect to the reduced density matrix of the ancilla qubit. If we choose $\delta_{a}=\delta_{a}=0$ and $|A\rangle=|0\rangle$ we arrive at the cloning transformation proposed by Bužek and Hillery [6], one example for the optimum symmetric and isotropic cloner.

As the requirements for the scalar products of the ancilla states for the optimum cloner can be met by using ancilla states of dimension 2 there is no better cloner using higherdimensional ancillas. We note that maximizing the global fidelity, defined by $F_{g}=\operatorname{Tr}\left[\left(\rho_{\psi} \otimes \rho_{\psi}\right) \rho_{1,2}\right]$, where $\rho_{1,2}$ denotes the total output density matrix, traced over the ancilla, leads to the same transformations (17) and (18).

\section{B. Universal cloning by teleportation}

So far we viewed the cloner as a machine that clones quantum states at a given location. There are, however, certain scenarios, especially in quantum communication and cryptography, where cloning is followed by further processing that may involve sending the two clones to two different locations. In these scenarios one may benefit from "nonlocal" cloning, which can be achieved via teleportation.

Suppose that a sender (Alice) is to transmit an imperfect copy of her qubit state to two receivers (Bob and Charlie); the three parties possess as a starting resource a particular entangled quantum state, but otherwise only classical communication is permitted from Alice to Bob and Charlie. This situation is essentially the three-party generalization of the well-known teleportation protocol [7], in which Alice can transmit any qubit state to Bob perfectly, provided that they share an entangled singlet state $\left|\Psi^{-}\right\rangle=1 / \sqrt{2}(|01\rangle-|10\rangle)$. In this protocol, Alice first performs a joint measurement of the state to be teleported $|\psi\rangle$ and her half of the singlet pair, the measurement being performed in the Bell basis 


$$
\begin{aligned}
& \left|\Psi^{ \pm}\right\rangle=\frac{1}{\sqrt{2}}(|01\rangle \pm|10\rangle) \\
& \left|\Phi^{ \pm}\right\rangle=\frac{1}{\sqrt{2}}(|00\rangle \pm|11\rangle) .
\end{aligned}
$$

Alice then sends a two-bit message to Bob indicating which of the four Bell states was measured. Bob can reconstitute $|\psi\rangle$ exactly from his half of the singlet if he performs the final action: if he receives the message " $\Psi^{-}$,', nothing; if " $\Psi^{+}$, ," rotate his qubit by $\sigma_{z}$; if ' $\Phi^{-}$,", rotate by $\sigma_{x}$; if " $\Phi^{+}$, ,' rotate by $\sigma_{y}$.

The same protocol, but applied to the particular threeparticle state

$$
\left|\Psi_{\text {clone }}\right\rangle=\sqrt{\frac{2}{3}}|100\rangle-\sqrt{\frac{1}{6}}|010\rangle-\sqrt{\frac{1}{6}}|001\rangle,
$$

results precisely in a Bužek-Hillery cloning from Alice to Bob and Charlie, provided that the results are averaged over the four possible measurement outcomes. (This averaging is not necessary in ordinary teleportation; we will explain in a moment what happens if the measurement outcomes are not averaged over in the present form of teleportation.) In $\left|\Psi_{\text {clone }}\right\rangle$, the first particle is possessed by Alice, the second by Bob, and the third by Charlie. (Of course, the state is symmetric with respect to Bob and Charlie.) The cloning is achieved by classical transmission in the sense that Alice need only broadcast the two-bit result of her Bell measurement to Bob and Charlie, with which they perform the same final action as in teleportation, in order for Bob and Charlie to possess Bužek-Hillery clones of Alice's original qubit state.

It is informative to formulate our imperfect teleportation in the language of quantum operations with which Nielsen and Caves have analyzed ordinary teleportation [10]. In this language the transformation from Alice's input state $\rho_{\psi}$ and Bob's (or Charlie's) output state $\rho_{o}^{i}$ conditional upon measurement outcome $i$ (unnormalized) is specified by the superoperator

$$
\rho_{o}^{i}=\sum_{j} A_{i j} \rho_{\psi} A_{i j}^{\dagger}
$$

The output density operator $\rho_{o}$ taking all measurement outcomes into account just requires the sum over all outcomes $i$ :

$$
\rho_{o}=\sum_{i, j} A_{i j} \rho_{\psi} A_{i j}^{\dagger}
$$

Completeness requires

$$
\sum_{i, j} A_{i j}^{\dagger} A_{i j}=1
$$

but if the sum is restricted to a particular $i$,

$$
\sum_{j} A_{i j}^{\dagger} A_{i j}=E_{i}
$$

where $E_{i}$ is the operator representing the measurement outcome $i$ in the positive-operator-valued theory of quantum measurement.

This representation may be related simply to the Blochvector picture. If we write the conditional output density operator as

$$
\rho_{o}^{i}=\frac{\operatorname{Pr}(i)}{2}\left(\mathbf{1}+\vec{s}_{o} \cdot \vec{\sigma}\right)
$$

where $\operatorname{Pr}(i)$ is the probability of measurement outcome $i$, then

$$
\operatorname{Pr}(i)=\frac{1}{2} \operatorname{Tr} E_{i}+\frac{1}{2} \sum_{\alpha} s_{\psi \alpha} \operatorname{Tr}\left(E_{i} \sigma_{\alpha}\right)
$$

and

$$
\begin{aligned}
\operatorname{Pr}(i) s_{o \beta}= & \frac{1}{2} \operatorname{Tr}\left(\sum_{j} A_{i j} A_{i j}^{\dagger} \sigma_{\beta}\right) \\
& +\frac{1}{2} \sum_{\alpha} s_{\psi \alpha} \operatorname{Tr}\left(\sum_{j} A_{i j} \sigma_{\alpha} A_{i j}^{\dagger} \sigma_{\beta}\right) .
\end{aligned}
$$

A straightforward calculation shows that for our imperfect teleportation, the $\Phi^{+}$and $\Phi^{-}$measurement outcomes are indistinguishable (i.e., are described by the same $A$ operators and therefore have the same probability of occurrence and leave the output qubit in the identical state). This is also true of the $\Psi^{+}$and $\Psi^{-}$outcomes. However, the $\Phi$ and $\Psi$ measurements are distinct. This is in contrast to perfect teleportation in which all four measurement outcomes lead to identical operations (just the trivial noiseless identity operator, in fact). For our case we find

$$
\begin{array}{ll}
A_{\Phi, 1}=\sqrt{\frac{2}{3}}\left(\begin{array}{cc}
\frac{1}{2} & 0 \\
0 & 1
\end{array}\right), & A_{\Phi, 2}=\sqrt{\frac{1}{6}}\left(\begin{array}{ll}
0 & 0 \\
1 & 0
\end{array}\right), \\
A_{\Psi, 1}=\sqrt{\frac{2}{3}}\left(\begin{array}{cc}
1 & 0 \\
0 & \frac{1}{2}
\end{array}\right) & A_{\Psi, 2}=\sqrt{\frac{1}{6}}\left(\begin{array}{ll}
0 & 1 \\
0 & 0
\end{array}\right),
\end{array}
$$

and

$$
E_{\Phi}=\left(\begin{array}{cc}
\frac{1}{3} & 0 \\
0 & \frac{2}{3}
\end{array}\right), \quad E_{\Psi}=\left(\begin{array}{cc}
\frac{2}{3} & 0 \\
0 & \frac{1}{3}
\end{array}\right)
$$

We note from Eq. (27) that the teleportation operation, keeping only the cases where the measurement outcome is a $\Phi$, resembles in some ways a "decay channel" in which the state is damped towards the $|1\rangle$-state fixed point. The cases where the measurement outcome is $\Psi$ behave identically except with $|0\rangle$ and $|1\rangle$ interchanged (from which the isotropy of the measurement-averaged operation emerges). However, it is incorrect to say that the total operation is obtained by selecting at random between the " $\Phi$ channel" and the " $\Psi$ channel" (although this is the way that [11] creates several interesting cloning transformations), because the $E_{i}$ operators are not proportional to the identity as they are in perfect teleportation. Unlike in the randomly selected channel, the 
probability of the measurement outcome depends on the input state; we find directly from Eq. (28) that

$$
\begin{aligned}
& \operatorname{Pr}(\Phi)=\frac{1}{3}\left\langle 0\left|\rho_{\psi}\right| 0\right\rangle+\frac{2}{3}\left\langle 1\left|\rho_{\psi}\right| 1\right\rangle, \\
& \operatorname{Pr}(\Psi)=\frac{2}{3}\left\langle 0\left|\rho_{\psi}\right| 0\right\rangle+\frac{1}{3}\left\langle 1\left|\rho_{\psi}\right| 1\right\rangle .
\end{aligned}
$$

Finally, we note that since the Bell measurements occupy a Hilbert space of at least two qubits [12], an open question is raised of whether good 1-to- $N$ cloning can be achieved by teleportation through an $(N+1)$-particle entangled state. In the simplest generalization of the above protocol the extra Hilbert space size would still be two qubits (since there would still just be one Bell measurement), but the optimal 1-to- $N$ cloner appears to require an ancilla with $O(N)$ qubits [13]; as Bužek has pointed out, this may well mean that this teleportation approach to cloning may not generalize to other cloning problems.

\section{STATE-DEPENDENT QUANTUM CLONERS}

Let us start this section with showing that in order to satisfy the isotropy requirement an ancilla system must be necessarily involved in the cloning transformation. This is proved in Sec. III A. In Sec. III B we drop the isotropy condition and investigate the case of a symmetric statedependent cloner in absence of ancilla. We will show that if we have some a priori knowledge about the input states the cloner can perform much better than the optimal universal one.

\section{A. Quantum cloner without ancilla}

From the general ansatz for the unitary transformation with an ancilla of arbitrary dimension we can draw conclusions about a quantum cloner without ancilla by replacing all states $|A\rangle,\left|B_{i}\right\rangle, \ldots$ on the right-hand sides of Eqs. (3) and (4) with the factor 1.

If we attempt to realize a symmetric and isotropic cloner we need to be able to fulfill the constraints (i)-(vii) where all scalar products of auxiliary states have to be replaced by 1 . We will show that this is not possible.

Here we only write down those four constraints that we need for our argument: (i) $|a|^{2}-|c|^{2}=|\widetilde{a}|^{2}-|\widetilde{c}|^{2}$, (ii) $|a|^{2}$ $-|c|^{2}=\operatorname{Re}\left[\tilde{b}_{1}^{*} a+\widetilde{a}^{*} b_{1}\right], \quad(\mathrm{v}) b_{2}^{*} a+c^{*} b_{1}=0$, and (vi) $\tilde{b}_{2}^{*} \tilde{a}+\widetilde{c}^{*} \widetilde{b}_{1}=0$. Remember that from (7) we have $\left|b_{1}\right|$ $=\left|b_{2}\right|=|b|$ and $\left|\widetilde{b}_{1}\right|=\left|\widetilde{b}_{2}\right|=|\tilde{b}|$. In order to fulfill constraints (v) and (vi) where both real and imaginary parts of the given sum have to vanish there are only these possibilities (for any choice of phases $\delta_{a}, \delta_{b_{i}}, \ldots$ ):

$$
(\mathrm{v}) \rightsquigarrow|b|=0 \text { or }|a|=|c|
$$

and

$$
\text { (vi) } \rightsquigarrow|\widetilde{b}|=0 \text { or }|\widetilde{a}|=|\widetilde{c}| \text {. }
$$

There are four possible combinations of these constraints: $|a|=|c|$ and $|\widetilde{a}|=|\widetilde{c}|,|a|=|c|$ and $|\widetilde{b}|=0,|b|=0$ and $|\widetilde{a}|$ $=|\widetilde{c}|$, and $|b|=0$ and $|\widetilde{b}|=0$. For the first three possibilities we find immediately from (i) and Eq. (11) that $\eta=0$, the trivial solution. For the last possibility we only need a glance at constraint (ii) to find $\eta=0$ as well. We thus conclude that it is impossible to build a symmetric isotropic quantum cloner without ancilla.

\section{B. Optimal state-dependent cloner}

In this subsection we answer the following question: Given two possible input states $|a\rangle$ and $|b\rangle$, where in general $\langle a \mid b\rangle \neq 0$, what is the optimal quantum cloner with respect to a global fidelity criterion? We suppose that the input qubit is prepared with the same probability in either state $|a\rangle$ or $|b\rangle$ and optimize the transformation as a function of their scalar product. The resulting optimal transformation will be therefore state dependent.

Two pure nonorthogonal states in a two-dimensional Hilbert space can be parametrized as

$$
\begin{aligned}
& |a\rangle=\cos \theta|0\rangle+\sin \theta|1\rangle, \\
& |b\rangle=\sin \theta|0\rangle+\cos \theta|1\rangle,
\end{aligned}
$$

where $\{|0\rangle,|1\rangle\}$ represents an orthonormal basis and $\theta$ $\in[0, \pi / 4]$. The set of the two input states can equivalently be specified by means of their scalar product $S=\langle a \mid b\rangle=\sin 2 \theta$.

Let us consider a unitary operator $U$ acting on $\mathcal{H}^{T}$ $=\mathcal{H}^{2} \otimes \mathcal{H}^{2}$ and define the final states $|\alpha\rangle$ and $|\beta\rangle$ as

$$
\begin{aligned}
& |\alpha\rangle=U|a\rangle|0\rangle, \\
& |\beta\rangle=U|b\rangle|0\rangle .
\end{aligned}
$$

Unitarity gives the following constraint on the scalar product of the final states:

$$
\langle\alpha \mid \beta\rangle=\langle a \mid b\rangle=\sin 2 \theta \equiv S
$$

As a criterion for optimality of the state-dependent cloner, we take the transformation that maximizes the global fidelity $F_{g}$ of both final states $|\alpha\rangle$ and $|\beta\rangle$ with respect to the perfect cloned states $|a a\rangle \equiv|a\rangle \otimes|a\rangle$ and $|b b\rangle \equiv|b\rangle \otimes|b\rangle$. The global fidelity is defined formally as

$$
F_{g}=\frac{1}{2}\left(|\langle\alpha \mid a a\rangle|^{2}+|\langle\beta \mid b b\rangle|^{2}\right) .
$$

We show in Appendix B that the above fidelity is maximized when the states $|\alpha\rangle$ and $|\beta\rangle$ lie in the two-dimensional space $\mathcal{H}_{a a, b b}$, which is spanned by vectors $\{|a a\rangle,|b b\rangle\}$.

Let us now maximize explicitly the value of the global fidelity (36). We can think about it in a geometrical way and define $\phi, \delta$, and $\gamma$ as the "angles"' between vectors $|a a\rangle$ and $|b b\rangle,|a a\rangle$ and $|\alpha\rangle$, and $|\alpha\rangle$ and $|\beta\rangle$, respectively. The global fidelity (36) then takes the form

$$
F_{g}=\frac{1}{2}\left[\cos ^{2} \delta+\cos ^{2}(\phi-\gamma-\delta)\right]
$$

and is thus maximized when the angle between $|a a\rangle$ and $|\alpha\rangle$ is equal to the angle between $|b b\rangle$ and $|\beta\rangle$, i.e., $\delta=\frac{1}{2}$ ( $\phi$ $-\gamma)$. The optimal situation thus corresponds to the maximal symmetry in the disposition of the vectors. 
As we know that $\phi=\arccos \left(\sin ^{2} 2 \theta\right)$ and from Eq. (35) $\gamma=\arccos (\sin 2 \theta)$, after little algebra we can write the optimal global fidelity as

$$
F_{g, o p t}=\frac{1}{4}\left(\sqrt{1+\sin ^{2} 2 \theta} \sqrt{1+\sin 2 \theta}+\cos 2 \theta \sqrt{1-\sin 2 \theta}\right)^{2} .
$$

The corresponding unitary transformation $U$ on the basis states $|00\rangle$ and $|10\rangle$ of the initial subspace of the fourdimensional Hilbert space of the two qubits is given by

$$
\begin{aligned}
& U|00\rangle=a|00\rangle+b(|01\rangle+|10\rangle)+c|11\rangle, \\
& U|10\rangle=c|00\rangle+b(|01\rangle+|10\rangle)+a|11\rangle,
\end{aligned}
$$

where

$$
\begin{gathered}
a=\frac{1}{\cos 2 \theta}[\cos \theta(P+Q \cos 2 \theta)-\sin \theta(P-Q \cos 2 \theta)], \\
b=\frac{1}{\cos 2 \theta} P \sin 2 \theta(\cos \theta-\sin \theta), \\
c=\frac{1}{\cos 2 \theta}[\cos \theta(P-Q \cos 2 \theta)-\sin \theta(P+Q \cos 2 \theta)],
\end{gathered}
$$

with

$$
\begin{gathered}
P=\frac{1}{2} \frac{\sqrt{1+\sin 2 \theta}}{\sqrt{1+\sin ^{2} 2 \theta}}, \\
Q=\frac{1}{2} \frac{\sqrt{1-\sin 2 \theta}}{\cos 2 \theta} .
\end{gathered}
$$

The transformation for $|\alpha\rangle$ and $|\beta\rangle$ can be readily derived from Eqs. (39) and (40). We can easily see that the transformation is symmetric, i.e., $\rho_{\alpha, 1}=\rho_{\alpha, 2}=\rho_{\alpha}$ for input state $|a\rangle$ and similarly for $|b\rangle$.

In order to compare the performance of the statedependent cloner with the universal one we calculate the local fidelity $F_{l}$ of each of the output copies with respect to the input one, generally defined as

$$
F_{l}=\operatorname{Tr}\left[\rho_{\alpha}|a\rangle\langle a|\right]
$$

For the above transformation we find

$$
\begin{gathered}
F_{l, 1}=\frac{1}{2}\left[1+\frac{\cos ^{2} 2 \theta}{\sqrt{1+\sin ^{2} 2 \theta}}+\frac{\sin ^{2} 2 \theta(1+\sin 2 \theta)}{1+\sin ^{2} 2 \theta}\right] \\
=\frac{1}{2}\left[1+\frac{1-S^{2}}{\sqrt{1+S^{2}}}+\frac{S^{2}(1+S)}{1+S^{2}}\right] .
\end{gathered}
$$

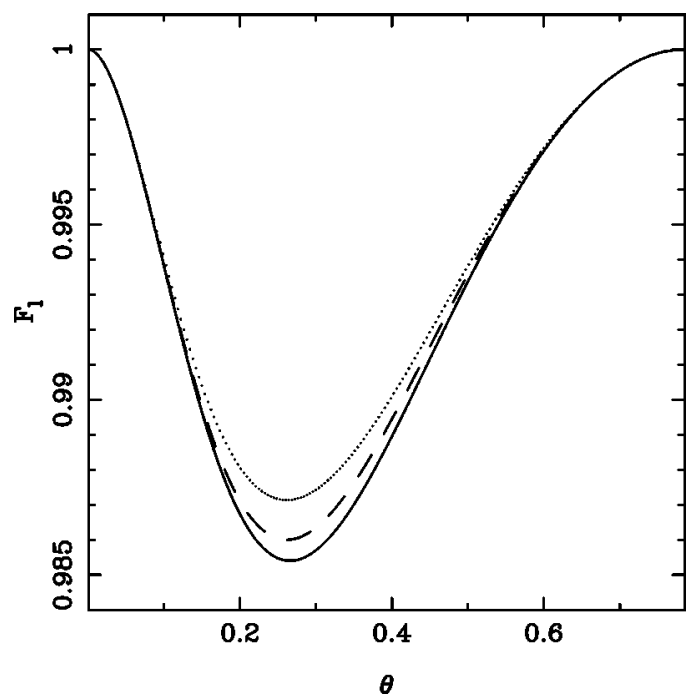

FIG. 1. Local fidelity of the state-dependent cloner as a function of $\theta$ : the solid line results from maximization of the global fidelity [see Eq. (47)], the dashed line corresponds to the local fidelity in the optimal eavesdropping scheme [given in Eq. (50)], and the dotted line is the optimal local fidelity [see Eq. (51)].

Due to the symmetry of the problem the same expression (47) is obtained for the fidelity of $\rho_{\beta}$ and it is plotted in Fig. 1. As we can see, the fidelity takes surprisingly high values in the whole range of $\theta$, well above the optimal value $5 / 6$ of the universal cloner.

Let us now examine the degree of entanglement that our "quasicloning" transformation has introduced in the system. An estimation of the degree of purity of the state is given by the modulus of the $\vec{s}$ vector in the Bloch sphere: The modulus is maximized to unity when the state is pure. In the case under consideration it takes the form

$$
|\vec{s}|=\sqrt{\frac{\sin ^{2} 2 \theta(1+\sin 2 \theta)^{2}}{\left(1+\sin ^{2} 2 \theta\right)^{2}}+\frac{\cos ^{2} 2 \theta}{1+\sin ^{2} 2 \theta}}
$$

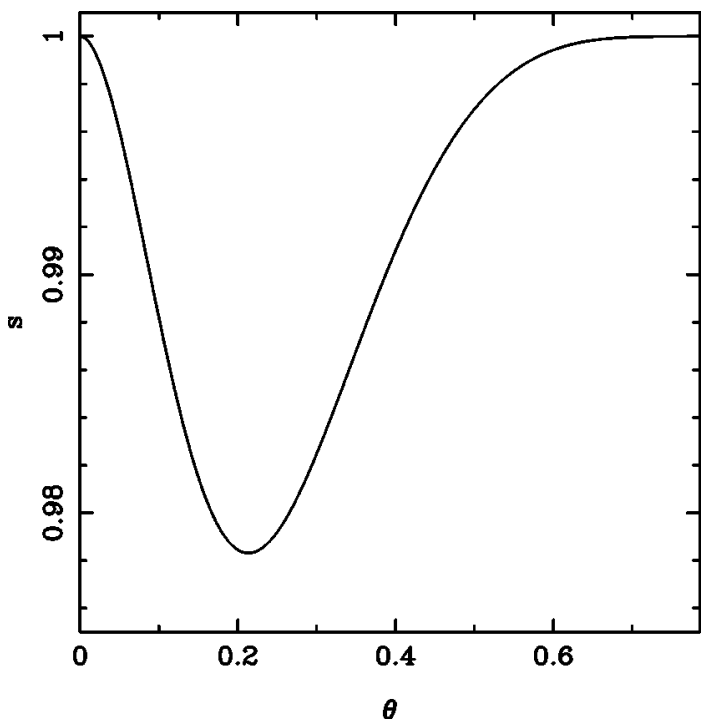

FIG. 2. Modulus of the Bloch vector $\vec{s}$ for the optimal statedependent cloner [see Eq. (48)]. 
and is plotted in Fig. 2. As we can see, the top of the vector $\vec{s}$ is always very close to the surface of the Bloch sphere for any value of $\theta$ and the degree of purity of the output state is therefore always fairly high. Notice that the length of the Bloch vector is always much bigger than the value $2 / 3$ of the optimal universal cloner. We also point out that in this case the Bloch vector is not only shrunk but also rotated by a state-dependent angle $\vartheta$, given by

$$
\vartheta=\arccos \left[\frac{1}{|\vec{s}|} \frac{\cos 2 \theta}{\sqrt{1+\sin ^{2} 2 \theta}}\right]-2 \theta .
$$

Perhaps the most important practical use for statedependent cloners is in the eavesdropping on some quantum cryptographic systems. For example, if the quantum key distribution protocol is based on two nonorthogonal states [5], the optimal state-dependent cloner can clone the qubit in transit between a sender and a receiver. The original qubit can then be re-sent to the receiver and the clone can stay with an eavesdropper who by measuring it can obtain some information about the bit value encoded in the original. The eavesdropper may consider storing the clone and delaying the actual measurement until any further public communication between the sender and the receiver takes place. This eavesdropping strategy, for instance, has been discussed recently in Ref. [11].

It should be noted, however, that eavesdropping via a direct cloning attempt is not the most advisable course of action for the eavesdropper if she wishes to be the most sur- reptitious. For that task, the eavesdropper's main concern is not in copying the quantum information, as embodied in the two nonorthogonal quantum states, but rather in optimizing the trade-off between the classical information made available to her versus the disturbance inflicted upon the original qubit $[8,9]$. The optimal solution to that problem leads to a one-parameter class of unitary interactions, the parameter being the degree of disturbance. It turns out that, regardless of the value of the parameter, the optimal unitary interaction there never matches that given in Eqs. (39)-(45).

Indeed this can be seen in a direct manner. The optimal eavesdropping strategy is quite similar to the scenario described above. The eavesdropper uses a probe system to interact with the in-transit qubit and then later performs a measurement on it (after all public discussion has ceased). Although it is not assumed, it turns out to be sufficient to take the probe system itself to be a single qubit $[8,9]$. In general, the final state of the probe will not be the same as that of the receiver's qubit: For instance, if the eavesdropper's available information is adjusted to vanish, then her probe will be left in its original state, which is completely independent of the sender's qubit's state. Nevertheless, in Appendix $\mathrm{C}$ it is shown that when the disturbance is adjusted so that the statistical distinguishability between the states of the eavesdropper's probe is identical to that of the final states of the receiver's qubit, then the optimal eavesdropping scheme is actually a quantum cloner. In that case, the local fidelity between input and output works out to be

$$
F_{l, 2}=\frac{1}{2}+\frac{\sqrt{2}}{4} \sqrt{\left(1-2 S^{2}+2 S^{3}+S^{4}\right)+\left(1-S^{2}\right) \sqrt{(1+S)\left(1-S+3 S^{2}+S^{3}\right)}} .
$$

The difference between this fidelity and $F_{l, 1}$ in Eq. (47) is only slight (they differ at most by 0.000651 when $S$ $=0.579$ 924; see Fig. 1), but this is enough to show that optimal cloning and optimal eavesdropping are two different tasks.

Similar results can be obtained for the four states in the Bennett-Brassard quantum cryptographic protocol [14]. Modifying the optimal eavesdropping scheme for that protocol in Ref. [15] into a quantum cloning device as above gives a local fidelity of 0.854 . Note that in the scenario of Bennett and Brassard we can restrict the input of a cloning machine; therefore, one would not want to use the universal BužekHillery cloner for the task of eavesdropping in the BennettBrassard protocol.

A more intriguing point, however, can be gleaned from noting that actually for all $S, F_{l, 2} \geqslant F_{l, 1}$. This implies that the optimal global quantum cloner is not optimized with respect to the local fidelity criterion: In the state-dependent case, the two criteria differ. In fact, the state-dependent cloner derived from optimal eavesdropping is still not the best with respect to the local fidelity criterion. For instance, in Appendix $\mathrm{C}$ it is shown that there is a still better state-dependent cloner for this criterion; it gives rise to a local fidelity given by

$$
\begin{aligned}
F_{l, 3}= & \frac{1}{2}+\frac{\sqrt{2}}{32 S}(1+S)\left(3-3 S+\sqrt{1-2 S+9 S^{2}}\right) \\
& \times \sqrt{-1+2 S+3 S^{2}+(1-S) \sqrt{1-2 S+9 S^{2}}} .
\end{aligned}
$$

Again, the difference between $F_{l, 3}$ and $F_{l, 2}$ is not large (the largest difference 0.001134 is attained when $S=1 / 2$; see Fig. 1 ), but it is enough to show that there are better cloners out there with respect to the local fidelity criterion. We have verified that $F_{l, 3}$ is indeed the optimal local fidelity for a state-dependent cloner as defined in Eqs. (39) and (40), but refrain from presenting the tedious calculations here. Ultimately, the disparity between Eqs. (47), (50), and (51) only points out the subtlety of the concept of "copying" quantum information: Given that it cannot be done ideally, there is no single sense in which it can be done in the best possible way.

Finally, let us note that in this subsection we have always considered qubits for the purpose of illustration, but we stress that the results hold for an arbitrary dimension of the input states. In this case we can rephrase our arguments in terms of the two-dimensional subspace spanned by the two input states and choose the same parametrization as given in 
Eq. (32) for the input states in such subspace. We can then derive the same conclusions as above.

\section{APPLICATION TO QUANTUM CAPACITY}

The optimal universal cloners, e.g., the Bužek-Hillery cloner, permit us to establish an upper bound on the quantum capacity of a depolarizing qubit channel. A simple $(1-\eta)$-depolarizing channel transmits a quantum state whose Bloch vector is shrunk by $\eta$, as above. The quantum capacity $Q(\eta)$ is the maximum rate at which $k$ qubits can be coded into $n$ qubits in such a way that the $k$ qubits can be recovered with high fidelity by the receiver, in the limit of $k$ and $n$ going to infinity. We can show that

$$
\begin{gathered}
Q=0, \quad \eta \leqslant \frac{2}{3} \\
Q(\eta) \leqslant 1-H_{2}\left(\frac{3}{4} \eta+\frac{1}{4}\right), \quad \eta>\frac{2}{3},
\end{gathered}
$$

where $H_{2}(x)=-x \log _{2} x-(1-x) \log _{2}(1-x)$ is the binary entropy function.

The second part of Eq. (52) is proved in $[16,17]$. The proof of $Q=0$ for $\eta=\frac{2}{3}$ follows from the universal cloning results above. Suppose the opposite, $Q\left(\eta=\frac{2}{3}\right)>0$; Sec. IV of [18] shows that this cannot be so: Consider the BužekHillery cloner inserted into a three-party Alice-Bob-Charlie communications protocol discussed above in Sec. II B. If Bob and Charlie were oblivious to each other's existence, they could both, by experiments conducted in concert with Alice, establish that the Alice-Bob channel and the AliceCharlie channel are both simple depolarizing channels with depolarization fractions $\eta=\frac{2}{3}$. If $Q\left(\frac{2}{3}\right)>0$ this would mean that Alice could, with suitable encoding, transmit a state to Bob and Charlie, both of whom could successfully decode it and obtain a high-fidelity copy of it. However, this violates the no-cloning theorem for quantum states [1]; thus it must be so that $Q\left(\frac{2}{3}\right)=0$. $Q=0$ for $\eta<\frac{2}{3}$ follows from the nondecreasing (as a function of $\eta$ ) property of $Q$ : If a lower $\eta$ gave a higher $Q$ then Alice could add noise herself to the signal thereby turning a high- $\eta$ channel into the supposedly better lower- $\eta$ channel.

The bound given in Eq. (52) is discontinuous at $\eta=\frac{2}{3}$. If we made the seemingly natural assumption that $Q$ is a continuous function of $\eta$, as is the channel capacity in the classical setting, then we can apply the methods introduced in [18] to show that $Q \leqslant 3 \eta-2$ for $\eta>\frac{2}{3}$. This would improve on Eq. (52) for a range of $\eta$ 's near $\frac{2}{3}$. Unfortunately, the continuity of $Q$ has proved surprisingly difficult to establish rigorously; this has finally been established [19] for a particular channel, the quantum erasure channel. The fact that the Bužek-Hillery cloner is proved to be optimal shows that no stronger bound on $Q$ for the depolarizing channel can be established by this reasoning and in fact no upper bound with a lower threshold is known, although there is also no evidence that the capacity of Eq. (52) can be attained. Thus this remains one of the many open questions in quantum information theory.

\section{ACKNOWLEDGMENTS}

We thank Vladimir Bužek and Nicolas Gisin for discussions. This work was supported in part by European TMR Research Network Grant No. ERB-4061PL95-1412 and a NATO collaborative research grant. D.P.V. and J.A.S. thank the Army Research Office for support. C.A.F. would like to thank the Lee A. DuBridge Foundation for financial support. $\mathrm{He}$ also acknowledges support from DARPA through the Quantum Information and Computing Institute administered by the U.S. Army Research Office. Part of this work was completed during the Elsag-Bailey-ISI Foundation research meeting on quantum computation.

\section{APPENDIX A: OPTIMIZATION FOR UNIVERSAL CLONER}

We want to maximize the function $\eta$ while the constraints (i) -(vii) as well as the unitarity constraints (5) and (6) are fulfilled. The independent variables are the absolute values of the coefficients $a, b_{i}, \ldots$, their phases, the absolute values of the scalar products of the ancilla states [two of these are already fixed via the symmetry condition (7)], and their phases, which we denote

$$
\langle A \mid C\rangle=|\langle A \mid C\rangle| e^{i \delta_{A C},}
$$

and accordingly for the other scalar products.

We impose the natural symmetry requirement on the general ansatz that the reduced density matrix of the two clones should not change under the exchange $|0\rangle \leftrightarrow|1\rangle$, i.e., the outcome should not depend on renaming the basis. This leads us immediately to

$$
|a|=|\widetilde{a}|, \quad\left|b_{i}\right|=\left|\widetilde{b}_{i}\right|, \quad|c|=|\tilde{c}|
$$

and the following restrictions for the scalar products of ancilla states from off-diagonal density matrix elements:

$$
\begin{gathered}
\left|\left\langle A \mid B_{i}\right\rangle\right|=\left|\left\langle\widetilde{A} \mid \widetilde{B_{i}}\right\rangle\right|, \\
\left|\left\langle B_{i} \mid C\right\rangle\right|=\left|\left\langle\widetilde{B_{i}} \mid \widetilde{C}\right\rangle\right|, \quad|\langle C \mid A\rangle|=|\langle\widetilde{C} \mid \widetilde{A}\rangle| .
\end{gathered}
$$

We also find that the phases $\delta_{\widetilde{A} \widetilde{B}_{i}}, \delta_{\widetilde{B_{i}} \widetilde{C}}, \delta_{\widetilde{C A} \widetilde{A}}$ can be expressed as functions of the phases $\delta_{A B_{i}}, \delta_{B_{i} C}, \delta_{C A}$ and $\delta_{a}, \delta_{b_{i}}, \ldots$.

We are using the method of Lagrange multipliers, where we have to solve the system of equations

$$
\begin{gathered}
\frac{\partial \eta}{\partial|a|}+\sum_{i=1}^{13} \lambda_{i} \frac{\partial \varphi_{i}}{\partial|a|}=0 \\
\frac{\partial \eta}{\partial|b|}+\sum_{i=1}^{13} \lambda_{i} \frac{\partial \varphi_{i}}{\partial|b|}=0, \ldots \quad \varphi_{i} \equiv 0, \quad i=1, \ldots, 13,
\end{gathered}
$$

where

$$
\eta=2|a|^{2}+2|b|^{2}-1,
$$

$\varphi_{i}$ denotes the constraints, and the Lagrange multipliers are $\lambda_{i}$. The order of the constraints that defines the Lagrange multiplier indices in later equations is taken to be 


$$
\begin{gathered}
\varphi_{1}=|a|^{2}+2|b|^{2}+|c|^{2}-1, \\
\varphi_{2}=2|a|^{2}+2|b|^{2}-1-\operatorname{Re}\left[\widetilde{b}_{1}^{*} a\left\langle\widetilde{B}_{1} \mid A\right\rangle+\widetilde{a}^{*} b_{1}\left\langle\widetilde{A} \mid B_{1}\right\rangle\right], \\
\varphi_{3}=\operatorname{Im}\left[\widetilde{b}_{1}^{*} a\left\langle\widetilde{B}_{1} \mid A\right\rangle+\widetilde{a}^{*} b_{1}\left\langle\widetilde{A} \mid B_{1}\right\rangle\right], \\
\varphi_{4}=b_{1}^{*} \widetilde{c}\left\langle B_{1} \mid \widetilde{C}\right\rangle+c^{*} \widetilde{b}_{1}\left\langle C \mid \widetilde{B}_{1}\right\rangle, \\
\varphi_{5}=b_{2}^{*} a\left\langle B_{2} \mid A\right\rangle+c^{*} b_{1}\left\langle C \mid B_{1}\right\rangle, \\
\varphi_{6}=\widetilde{b}_{2}^{*} \widetilde{a}\left\langle\widetilde{B}_{2} \mid \widetilde{A}\right\rangle+\widetilde{c}^{*} \widetilde{b}_{1}\left\langle\widetilde{C} \mid \widetilde{B}_{1}\right\rangle, \\
\varphi_{7}=\widetilde{c} * a\langle\widetilde{C} \mid A\rangle-\widetilde{a}^{*} c\langle\widetilde{A} \mid C\rangle, \\
\varphi_{8}=a^{*}\langle A \mid \widetilde{C}\rangle+b_{2}^{*} \widetilde{b_{1}}\left\langle B_{2} \mid \widetilde{B_{1}}\right\rangle+b_{1}^{*} \widetilde{b_{2}}\left\langle B_{1} \mid \widetilde{B_{2}}\right\rangle \\
+c^{*} \widetilde{a}\langle C \mid \widetilde{A}\rangle \\
\varphi_{9,10,11,12,13}=\varphi_{2,3,4,5,6} \text { with } 1 \leftrightarrow 2 .
\end{gathered}
$$

In solving this system of equations we can use some knowledge about the coefficients. We know from constraint (ii) and Eq. (A2) that both $|a|$ and $|b|$ cannot take the value 0 because otherwise $\eta=0$, the trivial solution.

Taking the partial derivative with respect to $|c|$ leads to

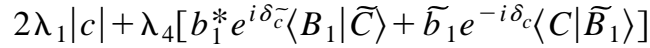

$$
\begin{aligned}
& +\lambda_{11}\left[b_{2}^{*} e^{i \delta_{c}}\left\langle B_{2} \mid \widetilde{C}\right\rangle+\widetilde{b}_{2} e^{-i \delta_{c}}\left\langle C \mid \widetilde{B_{2}}\right\rangle\right] \\
& +\lambda_{5} b_{1} e^{-i \delta_{c}}\left\langle C \mid B_{1}\right\rangle+\lambda_{12} b_{2} e^{-i \delta_{c}}\left\langle C \mid B_{2}\right\rangle
\end{aligned}
$$

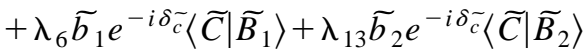

$$
\begin{aligned}
& +2 \lambda_{8} a^{*} e^{i \delta \tilde{c}}\langle A \mid \widetilde{C}\rangle=0,
\end{aligned}
$$

where we have already eliminated $\langle\widetilde{A} \mid C\rangle$ by inserting $\varphi_{7}$ $\equiv 0$ into $\varphi_{8}$. From the derivatives with respect to $\left|\left\langle B_{i} \mid \widetilde{C}\right\rangle\right|$, $\left|\left\langle C \mid \widetilde{B_{i}}\right\rangle\right|,\left|\left\langle C \mid B_{i}\right\rangle\right|,\left|\left\langle\widetilde{C} \mid \widetilde{B_{i}}\right\rangle\right|$, and $|\langle A \mid \widetilde{C}\rangle|$ we arrive (after dividing through phase factors) at

$$
\lambda_{j}|b||c|=0 \text { with } j=4,5,6,11,12,13
$$

and

$$
\lambda_{8}|a||c|=0
$$

After multiplying Eq. (A7) by $|c|$ we find

$$
\lambda_{1}|c|^{2}=0 \text {. }
$$

In the same way we use the equations resulting from differentiating with respect to $|a|,\left|\left\langle\widetilde{B_{i}} \mid A\right\rangle\right|,\left|\left\langle\widetilde{A} \mid B_{i}\right\rangle\right|,\left|\left\langle B_{i} \mid A\right\rangle\right|$, $\left|\left\langle\widetilde{B_{i}} \mid \widetilde{A}\right\rangle\right|$ and $|\langle A \mid \widetilde{C}\rangle|$ and get

$$
2|a|+\lambda_{1}|a|+2 \lambda_{2}|a|+2 \lambda_{9}|a|=0 .
$$

Multiplying this with $|c|^{2}$ and using Eq. (A10) we conclude that, since $|a| \neq 0$, either $\lambda_{2}+\lambda_{9}=-1$ or $|c|=0$.
We will now show that $\lambda_{2}+\lambda_{9}=-1$ corresponds to a minimum of $\eta$, i.e., $\eta=0$. From the derivatives with respect to $\left|\left\langle\widetilde{B_{1}} \mid A\right\rangle\right|$ and $\left|\left\langle\widetilde{B_{2}} \mid A\right\rangle\right|$ we find, after dividing through $|a||b|$,

$$
\begin{aligned}
& -\lambda_{2} \cos \left(\delta_{a}-\delta_{\widetilde{b}_{1}}+\delta_{\widetilde{B}_{1} A}\right)+\lambda_{3} \sin \left(\delta_{a}-\delta_{\widetilde{b}_{1}}+\delta_{\widetilde{B}_{1} A}\right)=0, \\
& -\lambda_{9} \cos \left(\delta_{a}-\delta_{\widetilde{b_{2}}}+\delta_{\widetilde{B}_{2} A}\right)+\lambda_{10} \sin \left(\delta_{a}-\delta_{\widetilde{b_{2}}}+\delta_{\widetilde{B}_{2} A}\right)=0
\end{aligned}
$$

and from the derivatives with respect to $\delta_{\widetilde{B}_{1} A}$ and $\delta_{\widetilde{B}_{2} A}$,

$$
\begin{aligned}
& \lambda_{2}\left|\left\langle\widetilde{B_{1}} \mid A\right\rangle\right| \sin \left(\delta_{a}-\delta_{\widetilde{b}_{1}}+\delta_{\widetilde{B}_{1} A}\right) \\
& +\lambda_{3}\left|\left\langle\widetilde{B_{1}} \mid A\right\rangle\right| \cos \left(\delta_{a}-\delta_{\widetilde{b_{1}}}+\delta_{\widetilde{B_{1}} A}\right)=0, \\
& \lambda_{9}\left|\left\langle\widetilde{B_{2}} \mid A\right\rangle\right| \sin \left(\delta_{a}-\delta_{\widetilde{b_{2}}}+\delta_{\widetilde{B_{2}} A}\right) \\
& \quad+\lambda_{10}\left|\left\langle\widetilde{B_{2}} \mid A\right\rangle\right| \cos \left(\delta_{a}-\delta_{\widetilde{b_{2}}}+\delta_{\widetilde{B_{2} A}}\right)=0 .
\end{aligned}
$$

If $\lambda_{2}+\lambda_{9}=-1$ then at least one of these two multipliers is not equal to zero. Let us assume that $\lambda_{2} \neq 0$. We multiply Eq. (A12) by $\cos \left(\delta_{a}-\delta_{\widetilde{b}_{1}}+\delta_{\widetilde{B}_{1} A}\right)$, obtaining

$$
\begin{gathered}
\lambda_{3} \cos \left(\delta_{a}-\delta_{\widetilde{b}_{1}}+\delta_{\widetilde{B}_{1} A}\right) \sin \left(\delta_{a}-\delta_{\widetilde{b}_{1}}+\delta_{\widetilde{B}_{1} A}\right) \\
=\lambda_{2} \cos ^{2}\left(\delta_{a}-\delta_{\widetilde{b}_{1}}+\delta_{\widetilde{B}_{1} A}\right) .
\end{gathered}
$$

Substituting Eq. (A16) into Eq. (A14) multiplied by $\sin \left(\delta_{a}\right.$ $\left.-\delta_{\widetilde{b_{1}}}+\delta_{\widetilde{B}_{1} A}\right)$, we obtain $\lambda_{2}\left|\left\langle\widetilde{B_{1}} \mid A\right\rangle\right|=0$, so that

$$
\left\langle\widetilde{B_{1}} \mid A\right\rangle=0 \text { if } c \neq 0
$$

The same reasoning in which tilded and untilded variables are interchanged leads to

$$
\left\langle\widetilde{A} \mid B_{1}\right\rangle=0 \text { if } c \neq 0
$$

Due to constraint (ii) this means that $\eta=0$.

If the assumption $\lambda_{2} \neq 0$ does not hold then $\lambda_{9} \neq 0$ and the same line of arguments leads to $\left\langle\widetilde{B_{2}} \mid A\right\rangle=\left\langle\widetilde{A} \mid B_{2}\right\rangle=0$ and also $\eta=0$. We have thus established $|c|=0$, and therefore $\eta=|a|^{2}$. We also notice that from $\varphi_{5}, \varphi_{12}$ and $\varphi_{6}, \varphi_{13}$ we need

$$
\left\langle B_{i} \mid A\right\rangle=\left\langle\widetilde{B_{i}} \mid \widetilde{A}\right\rangle=0
$$

Now $\eta$ is maximized by maximizing $|a|$, which can be easily achieved using constraint (ii) and the normalization condition:

$$
\begin{aligned}
\text { (ii) } \rightsquigarrow|a|^{2} & =\operatorname{Re}\left[\widetilde{b}_{1}^{*} a\left\langle\widetilde{B_{1}} \mid A\right\rangle+\widetilde{a}^{*} b_{1}\left\langle\widetilde{A} \mid B_{1}\right\rangle\right] \\
& =|a||b| \operatorname{Re}\left[e^{i\left(\delta_{a}-\delta_{b_{1}}\right)}\left\langle\widetilde{B_{1}} \mid A\right\rangle+e^{i\left(\delta_{b_{1}}-\delta_{a}\right)}\left\langle\widetilde{A} \mid B_{1}\right\rangle\right] \\
& =|a||b| \xi
\end{aligned}
$$

or 


$$
|a|^{2}=1-\frac{2}{2+\xi^{2}} .
$$

So $|a|^{2}$ is maximized for the maximum value of $\xi^{2}$, which is $\xi^{2}=2^{2}$. This leads to

$$
|a|=\sqrt{\frac{2}{3}}, \quad|b|=\sqrt{\frac{1}{6}} .
$$

We can meet the maximum of $\xi$ by choosing

$$
\left\langle\widetilde{A} \mid B_{i}\right\rangle=1, \quad\left\langle\widetilde{B_{i}} \mid A\right\rangle=1
$$

and

$$
\delta_{a}=\delta_{\widetilde{b}_{i}}, \quad \delta_{\widetilde{a}}=\delta_{b_{i}} .
$$

Collecting our information about the coefficients and scalar products, the class of optimal unitary transformations is given by

$$
\begin{aligned}
U|0\rangle|0\rangle|X\rangle= & \sqrt{\frac{2}{3}} e^{i \delta_{a}}|00\rangle|A\rangle \\
& +\sqrt{\frac{1}{6}} e^{i \delta_{a}^{\tilde{a}}(|01\rangle+|10\rangle)\left|A_{\perp}\right\rangle,} \\
U|1\rangle|0\rangle|X\rangle= & \sqrt{\frac{2}{3}} e^{i \delta_{a}^{-}}|11\rangle\left|A_{\perp}\right\rangle \\
& +\sqrt{\frac{1}{6}} e^{i \delta_{a}(|01\rangle+|10\rangle)|A\rangle,}
\end{aligned}
$$

where $\left\langle A \mid A_{\perp}\right\rangle=0$.

\section{APPENDIX B: OPTIMIZATION FOR STATE-DEPENDENT CLONER}

Let us assume that $|\alpha\rangle$ and $|\beta\rangle$ have some contribution that does not lie in $\mathcal{H}_{a a, b b}$. Then we can write explicitly the form of $|\alpha\rangle$ and $|\beta\rangle$

$$
\begin{aligned}
& |\alpha\rangle=a_{0}|a a\rangle+b_{0}|b b\rangle+c_{0}\left|C_{0}\right\rangle, \\
& |\beta\rangle=a_{1}|a a\rangle+b_{1}|b b\rangle+c_{1}\left|C_{1}\right\rangle,
\end{aligned}
$$

where vectors $\left|C_{0}\right\rangle$ and $\left|C_{1}\right\rangle$ are normalized and lie in the subspace orthogonal to $\mathcal{H}_{a a, b b}$. The unitarity of the transformation imposes the constraints

$$
\begin{aligned}
\varphi_{1}= & \operatorname{Re}\left[a_{0}^{*} a_{1}+b_{0}^{*} b_{1}+S^{2}\left(a_{0}^{*} b_{1}+b_{0}^{*} a_{1}\right)+c_{0}^{*} c_{1}\left\langle C_{0} \mid C_{1}\right\rangle\right] \\
& -S=0, \\
\varphi_{2}= & \operatorname{Im}\left[a_{0}^{*} a_{1}+b_{0}^{*} b_{1}+S^{2}\left(a_{0}^{*} b_{1}+b_{0}^{*} a_{1}\right)+c_{0}^{*} c_{1}\left\langle C_{0} \mid C_{1}\right\rangle\right] \\
= & 0, \\
& \varphi_{3}=\left|a_{0}\right|^{2}+\left|b_{0}\right|^{2}+2 S^{2} \operatorname{Re}\left[a_{0}^{*} b_{0}\right]+\left|c_{0}\right|^{2}-1=0, \\
& \varphi_{4}=\left|a_{1}\right|^{2}+\left|b_{1}\right|^{2}+2 S^{2} \operatorname{Re}\left[a_{1}^{*} b_{1}\right]+\left|c_{1}\right|^{2}-1=0,
\end{aligned}
$$

where $S$ is defined in Eq. (35).
The global fidelity is given by

$$
F_{g}=\frac{1}{2}\left(\left|a_{0}+b_{0} S^{2}\right|^{2}+\left|b_{1}+a_{1} S^{2}\right|^{2}\right)
$$

Inserting constraints $\varphi_{3}$ and $\varphi_{4}$ into Eq. (B7) yields

$$
F_{g}=\frac{1}{2}\left[2-\left(1-S^{4}\right)\left(\left|a_{1}\right|^{2}+\left|b_{0}\right|^{2}\right)-\left(\left|c_{0}\right|^{2}+\left|c_{1}\right|^{2}\right)\right] .
$$

We can now use the method of Lagrange multipliers for the remaining two constraints, which gives the system of equations

$$
\begin{aligned}
& \frac{\partial F_{g}}{\partial\left|a_{0}\right|}+\sum_{i=1}^{2} \lambda_{i} \frac{\partial \varphi_{i}}{\partial\left|a_{0}\right|}=0, \\
& \frac{\partial F_{g}}{\partial\left|b_{0}\right|}+\sum_{i=1}^{2} \lambda_{i} \frac{\partial \varphi_{i}}{\partial\left|b_{0}\right|}=0,
\end{aligned}
$$

etc. Let us concentrate on the equations where we differentiate with respect to the parameters $c_{0}, c_{1}$, and $\left\langle C_{0} \mid C_{1}\right\rangle$. Without loss of generality, we can consider $c_{0}$ and $\left\langle C_{0} \mid C_{1}\right\rangle$ real, while $c_{1}$ must be taken to be in general complex $\left(c_{1}\right.$ $\left.=\left|c_{1}\right| e^{i \delta}\right)$. The corresponding equations (obtained by differentiating with respect to $c_{0},\left|c_{1}\right|$, and $\left\langle C_{0} \mid C_{1}\right\rangle$, respectively) give

$$
\begin{gathered}
-c_{0}+\lambda_{1} \operatorname{Re}\left[\left|c_{1}\right| e^{i \delta}\left\langle C_{0} \mid C_{1}\right\rangle\right]+\lambda_{2} \operatorname{Im}\left[\left|c_{1}\right| e^{i \delta}\left\langle C_{0} \mid C_{1}\right\rangle\right]=0, \\
-\left|c_{1}\right|+\lambda_{1} \operatorname{Re}\left[c_{0} e^{i \delta}\left\langle C_{0} \mid C_{1}\right\rangle\right]+\lambda_{2} \operatorname{Im}\left[c_{0} e^{i \delta}\left\langle C_{0} \mid C_{1}\right\rangle\right]=0, \\
\lambda_{1} \operatorname{Re}\left[c_{0}\left|c_{1}\right| e^{i \delta}\right]+\lambda_{2} \operatorname{Im}\left[c_{0}\left|c_{1}\right| e^{i \delta}\right]=0 .
\end{gathered}
$$

After multiplying Eq. (B9) by $c_{0}$, Eq. (B10) by $\left|c_{1}\right|$, and Eq. (B11) by $\left\langle C_{0} \mid C_{1}\right\rangle$ and inserting the last equation into the other two we find $c_{0}=\left|c_{1}\right|=0$. We can therefore conclude that $|\alpha\rangle$ and $|\beta\rangle$ lie in $\mathcal{H}_{a a, b b}$.

\section{APPENDIX C: STATE-DEPENDENT CLONERS FROM EAVESDROPPING}

We take as our starting point for these calculations the development in Refs. [8] and [9] just at the point where the eavesdropper's probe is restricted to consist of a single qubit, i.e., we take $\sin \lambda=0$ in those references. This leaves a twoparameter family of unitary interactions to be considered. (Note that we shall interchange the symbols $\alpha$ and $\theta$ used in Refs. [8] and [9] so as to be consistent with the notation of the present paper.)

With this much given, suppose we label the receiver's state for his qubit after the eavesdropping interaction by $\rho_{a}^{\mathrm{A}}$ or $\rho_{b}^{\mathrm{A}}$, depending upon whether the sender sent state $|a\rangle$ or $|b\rangle$. Similarly suppose we label the eavesdropper's probe states by $\rho_{a}^{E}$ or $\rho_{b}^{E}$. Then we have from Eqs. (86)-(91) and (98)-(100) of Ref. [9] that the matrix elements for these operators will be 


$$
\begin{gathered}
\left(\hat{\boldsymbol{\rho}}_{a}^{E}\right)_{00}=\frac{1}{2}(1+\cos 2 \theta \cos 2 \phi), \\
\left(\hat{\boldsymbol{\rho}}_{a}^{E}\right)_{01}=\frac{1}{4}\left[(\cos \theta-\sin \theta)^{2} \sin 2(\phi-\alpha)\right. \\
\left.+(\cos \theta+\sin \theta)^{2} \sin 2(\phi+\alpha)\right], \\
\left(\hat{\rho}_{a}^{E}\right)_{11}=\frac{1}{2}(1-\cos 2 \theta \cos 2 \phi)
\end{gathered}
$$

and

$$
\begin{gathered}
\left(\hat{\rho}_{a}^{A}\right)_{00}=\cos ^{2} \theta \cos ^{2} \alpha+\sin ^{2} \theta \sin ^{2} \alpha, \\
\left(\hat{\rho}_{a}^{A}\right)_{01}=\cos \theta \sin \theta \sin 2 \phi \cos 2 \alpha+\frac{1}{2} \cos 2 \phi \sin 2 \alpha, \\
\left(\hat{\rho}_{0}^{A}\right)_{11}=\sin ^{2} \theta \cos ^{2} \alpha+\cos ^{2} \theta \sin ^{2} \alpha .
\end{gathered}
$$

Hermiticity determines the remainder of the matrix elements. The matrix elements for $\hat{\rho}_{b}^{E}$ and $\hat{\rho}_{b}^{A}$ are given by the same expressions, except with $\cos \theta$ and $\sin \theta$ interchanged. With this interaction, the fidelity between the sender's and receiver's states, i.e., $1-D$ in Eq. (33) of Ref. [8] and $1-D(U)$ in Eq. (101) of Ref. [9], is given by

$$
\begin{aligned}
F(\alpha, \phi) \equiv & \left\langle a\left|\rho_{a}^{A}\right| a\right\rangle=\left\langle b\left|\rho_{b}^{A}\right| b\right\rangle \\
= & \cos ^{2} \alpha+\frac{1}{2} S \cos 2 \phi \sin 2 \alpha \\
& -\frac{1}{2} S^{2}(1-\sin 2 \phi) \cos 2 \alpha .
\end{aligned}
$$

Now it is shown in Refs. [8] and [9] that if this interaction is to be one for optimizing the trade-off between the eavesdropper's information and the fidelity between the sender's and receiver's quantum states, then $\alpha$ and $\phi$ must satisfy the relation

$$
\tan 2 \alpha=\frac{S \cos 2 \phi}{1-S^{2}(1-\sin 2 \phi)}
$$

[See Eq. (52) in Ref. [8] and Eq. (108) in Ref. [9].] On the other hand, in order for the optimal eavesdropping solution to also be a quantum cloner, it must be the case that $\hat{\rho}_{a}^{\mathrm{E}}$ $=\hat{\rho}_{a}^{A}$ and $\hat{\rho}_{b}^{E}=\hat{\rho}_{b}^{A}$. A little algebra applied to Eqs. (C1)-(C6) shows that this can occur only when $\cos 2 \phi=\cos 2 \alpha$. Hence, if there is not to be an inconsistency with the constraint given by Eq. (C9), then it must be the case that the parameter $x$ $\equiv \sin 2 \phi$ is such that it satisfies

$$
\left(S+S^{2}\right) x^{2}+\left(1-S^{2}\right) x-S=0 .
$$

Solving this quadratic equation and inserting the result into Eq. (C8) gives the fidelity $F_{l, 2}$ of Eq. (50).

As stated in Sec. III B, this discussion can be expanded to produce a quantum cloner still better with respect to the "local" fidelity criterion than the one just found. We simply set $\phi=\alpha$ in the interaction above and ignore the constraint (C9) that the interaction lead to optimal eavesdropping. With this, Eq. (C8) reduces to

$$
F(\phi)=\frac{1}{2}+\frac{1}{2}(1+S)\left((1-S) \cos 2 \phi+\frac{1}{2} S \sin 4 \phi\right) .
$$

This expression is maximized when

$$
\sin 2 \phi=\frac{1}{4 S}\left(-1+S+\sqrt{1-2 S+9 S^{2}}\right) .
$$

Inserting this particular value for $\sin 2 \phi$ into Eq. (C11) gives the expression $F_{l, 3}$ reported in Eq. (51).
[1] W. K. Wootters and W. H. Zurek, Nature (London) 299, 802 (1982).

[2] D. Dieks, Phys. Lett. 92A, 271 (1982).

[3] H. P. Yuen, Phys. Lett. 113A, 405 (1986).

[4] H. Barnum, C. M. Caves, C. A. Fuchs, R. Jozsa, and B. Schumacher, Phys. Rev. Lett. 76, 2818 (1996).

[5] C. H. Bennett, Phys. Rev. Lett. 68, 3121 (1992).

[6] V. Bužek and M. Hillery, Phys. Rev. A 54, 1844 (1996).

[7] C. H. Bennett, G. Brassard, S. Popescu, B. Schumacher, J. Smolin, and W. K. Wootters, Phys. Rev. Lett. 76, 722 (1996).

[8] C. A. Fuchs and A. Peres, Phys. Rev. A 53, 2038 (1996).

[9] C. A. Fuchs, Fortschr. Phys. (to be published); e-print quant-ph/9611010.

[10] M. A. Nielsen and C. M. Caves, Phys. Rev. A 55, 2547 (1997); e-print quant-ph/9608001.

[11] N. Gisin and B. Huttner, Phys. Lett. A 228, 13 (1997).
[12] G. Brassard, S. L. Braunstein, and R. Cleve, Physica D (to be published).

[13] N. Gisin (privte communication).

[14] C. H. Bennett and G. Brassard, in Proceedings of the IEEE International Conference on Computer, Systems, and Signal Processing, Bangalore, India (IEEE, New York, 1984), pp. $175-179$.

[15] C. A. Fuchs, N. Gisin, R. B. Griffiths, C.-S. Niu, and A. Peres, Phys. Rev. A 56, 1163 (1997); e-print quant-ph/9701039.

[16] E. Rains, e-print quant-ph/9707002.

[17] V. Vedral and M. B. Plenio, e-print quant-ph/9707035.

[18] C. H. Bennett, D. P. DiVincenzo, J. A. Smolin, and W. K. Wootters, Phys. Rev. A 54, 3824 (1996); e-print quant-ph/9604024.

[19] H. Barnum, J. A. Smolin, and B. M. Terhal, e-print quant-ph/ 9711032. 